\title{
A survey on the incidence of Prototheca mastitis in dairy herds in Lublin province, Poland
}

\author{
Tomasz Jagielski, ${ }^{1 *}$ Katarzyna Roeske, ${ }^{1}$ Zofia Bakuła, ${ }^{1}$ Tomasz Piech, ${ }^{2}$ Łukasz Wlazło, ${ }^{3}$ Mariola Bochniarz, ${ }^{2}$ \\ Piotr Woch, ${ }^{4}$ and Henryk Krukowski ${ }^{3}$ \\ ${ }^{1}$ Department of Applied Microbiology, Institute of Microbiology, Faculty of Biology, University of Warsaw, I. Miecznikowa 1, 02-096 Warsaw, Poland \\ ${ }^{2}$ Department and Clinic of Animal Reproduction, Faculty of Veterinary Medicine, University of Life Sciences in Lublin, Głęboka 30, 20-612 Lublin, \\ Poland \\ ${ }^{3}$ Department of Animal and Environmental Hygiene, University of Life Sciences in Lublin, Akademicka 13, 20-950 Lublin, Poland \\ ${ }^{4}$ Regional Agrochemical Station in Lublin, Sławinkowska 5, 20-810 Lublin, Poland
}

\section{ABSTRACT}

Prototheca mastitis has recently become an emerging disease; although its incidence is increasing steadily, its epidemiology remains largely understudied. The aim of this work was to investigate the prevalence of Prototheca spp. in dairy cows and their environment in Lublin province, covering most of southeastern Poland. Between December 2015 and July 2016, a total of 172 milking cows from 10 dairy farms were inspected for mastitis using clinical examination and the California Mastitis Test (CMT). Quarter milk samples (QMS, n $=179)$ and body site swabs $(\mathrm{n}=151)$ from CMTpositive cows were collected for microbiological culture. In addition, we evaluated QMS and body site swabs from 23 healthy cows, along with 91 environmental samples. Of 100 CMT-positive cows, 71 had at least one QMS positive for microbial growth. In 8 (11.3\%) of these cows, originating from 7 dairy farms, Prototheca spp. were cultured. The average somatic cell count of the Prototheca-containing milk was $4.02 \times 10^{6}$ cells/ $\mathrm{mL}$ compared with $0.13 \times 10^{6}$ cells $/ \mathrm{mL}$ of the Prototheca-free milk (collected from control animals). No significant differences were observed between mastitis and control cows with respect to counts of total white blood cells, lymphocytes, neutrophils, and eosinophils. Half of the cows with Prototheca spp. in their milk did not yield the algae from other anatomical sites. Eight cows were negative for the presence of Prototheca spp. in their milk but positive for the algae in swabs from anatomical sites. Among the environmental sources that were positive for Prototheca growth were watering

Received August 3, 2018.

Accepted September 21, 2018.

*Corresponding author: t.jagielski@biol.uw.edu.pl troughs, manure, feed, and mud. All (45) Prototheca isolates recovered in this study were subjected to species- and genotype-level molecular identification. All QMS and most of the animal swabs (90\%) yielded Prototheca zopfii genotype (gen.) 2. Of the animal samples, P. zopfii gen. 1 and Prototheca blaschkeae were isolated only from feces and rectum. Environmental samples grew either P. zopfii gen. $2(67 \%)$ or P. zopfii gen. 1 (33\%). This study demonstrates that P. zopfii gen. 2 is the third most common pathogen of mastitis in cattle in southeast Poland, with an overall incidence of $4.6 \%$. Finding Prototheca spp., including P. zopfii gen. 1 and 2 and $P$. blaschkeae, in stool and rectal swabs from healthy animals may suggest their role as nonpathogenic microflora of bovine gut.

Key words: Prototheca spp., dairy herd, (sub)clinical mastitis, Poland

\section{INTRODUCTION}

Bovine mastitis, defined as an inflammation of the mammary gland, is the most prevalent and devastating disease in dairy cattle worldwide. It is also the most expensive problem faced by dairy farm owners, with the annual costs of US $\$ 2$ billion in the United States (Viguier et al., 2009). Economic losses are incurred either directly, through reduced milk production and premature culling of affected animals, or indirectly, from treatment and veterinary care expenses. Mastitis exists in 2 forms - clinical and subclinical. Clinical mastitis $(\mathbf{C M})$ is readily detectable due to the presence of visible changes in the udder (swelling, tenderness, hardness) and milk characteristics (wateriness, discoloration, clots, flakes), whereas subclinical mastitis (SCM) causes none or very few noticeable symptoms of inflammation and is detected by an elevated SCC in the produced milk. Subclinical mastitis is up to 40 times more frequent in dairy herds than CM (Seegers 
et al., 2003). The asymptomatic and latent course of SCM, with its tendency for chronicity, makes this form of mastitis much more epidemiologically hazardous and much less amenable to therapeutic intervention. Consequently, SCM imposes the greatest overall financial burden on the milk industry.

The etiological spectrum of bovine mastitis is wide and includes viruses; bacteria, with streptococci, staphylococci, and enterobacteria as the predominant pathogens; fungi, mostly yeasts; and achlorophyllous, yeast-like algae of the genus Prototheca (De Vliegher et al., 2012). The importance of the latter as causative agents of mastitis has long been underestimated, because of their misidentification as yeasts or dismissal as contaminating, saprophytic microflora. Prototheca algae were first implicated in bovine mastitis in 1952 (Lerche, 1952). Since then, protothecal mastitis has been recognized across the world, in countries such as the United States (Anderson and Walker, 1988), Canada (Pieper et al., 2012), Belgium (Lagneau, 1996), Denmark (Aalbaek et al., 1998), Germany (Möller et al., 2007), Italy (Buzzini et al., 2004), Brazil (Bueno et al., 2006), and Japan (Osumi et al., 2008). Over the last 2 decades, protothecal mastitis has become an emerging disease whose incidence is increasing steadily but whose epidemiology remains largely understudied. Additionally, given its inherent resistance to conventional antimicrobial therapies (Jagielski et al., 2012, 2017a), Prototheca infections pose a serious threat to the dairy sector.

Among the 8 Prototheca species currently recognized, 2 have been involved in mastitis events in cows: Prototheca zopfii and Prototheca blaschkeae, with the former being responsible for the vast majority of reported cases. Interestingly, of the $2 P$. zopfii genotypes (1 and 2), only $P$. zopfii genotype (gen.) 2 has been identified as causing the disease (Möller et al., 2007; Marques et al., 2008; Osumi et al., 2008; Ricchi et al., 2010; Jagielski et al., 2011). Still, P. zopfii gen. 1 has pathogenic potential, because it was shown to induce subclinical mastitis in a cow upon experimental infection (Ito et al., 2011).

In Poland, the occurrence of mastitis due to Prototheca algae was demonstrated for the first time in the early 2000s (Malinowski et al., 2002). Since then, cases of protothecal mastitis have been reported in different parts of the country. However, a prospective, large-sample, cross-country or cross-regional study on the incidence of Prototheca mastitis has never been performed in Poland. Therefore, the aim of this work was to evaluate the prevalence of Prototheca spp. in dairy cows and their environment in Lublin province, covering most of the southeast of Poland.

\section{MATERIALS AND METHODS}

\section{Dairy Farms and Herds}

Ten dairy farms (I to X), located in the Lublin province, Poland, housing herds varying in size from 12 to 806 cows, were inspected and sampled between December 2015 and July 2016 (Figure 1). All farms had reported mastitis events in at least 2 mo before the survey, according to the local veterinary records. A 25-point questionnaire was developed and tested to collect basic information on the dairy farms and herds, in a standardized form (Supplemental Table S1; https: //doi.org/10.3168/jds.2018-15495).

\section{Sampling Procedures}

Quarter milk samples (QMS) were collected from all lactating cows (if not being treated or during their pre- and postpartum periods) in herds up to 30 animals in total, or from 30 lactating cows, randomly selected, in herds whose size exceeded that number. Before milk collection, each cow was clinically examined, with special attention paid to the udder. A careful palpation of the udder was performed to detect any inflammatory swelling, fibrosis, induration, and other clinical signs. Premilking milk samples $(\sim 2 \mathrm{~mL})$ from each udder fraction were evaluated visually for clotting, color changes, and consistency. This was done in Schalm paddle wells, just before performing the California Mastitis Test (CMT; Mastirapid, Vetoquinol Biowet, Poland). The CMT results were scored as negative $(0)$, trace $(\mathrm{T})$, weak positive $(1+)$, distinctive positive $(2+)$, or strong positive (3+), as proposed earlier (Quinn et al., 2002). A CMT-positive (score of $\mathrm{T}$ or above) quarter milk was sampled for culture. The CMT-positive QMS were also tested for SCC by using an automated cell counter (Fossomatic 5000; Foss Electric, Hillerød, Denmark), as described previously (Gonzalo et al., 1993).

A mastitic cow was defined as any cow with an SCC $>200 \times 10^{3}$ cells $/ \mathrm{mL}$ (Hiitiö et al., 2017, Kirkpatrick and Olson, 2015). Clinical mastitis was determined based on apparent changes in milk (mild), signs of inflammation in the udder (moderate), and generalized clinical symptoms (severe). Subclinical mastitis was assessed using the CMT (Amer et al., 2018).

Diagnosis of mastitis required $\geq 1$ QMS positive for microbial growth. If 2 or more QMS from the same cow yielded different pathogens, 2 (or more) distinct mastitis infections were considered. If more than one pathogen was isolated from a single QMS, a mixed etiology was considered, with both organisms causal. Cows without mastitis were referred to as control animals. From each dairy herd, 2 (herds II, IV-IX) or 3 (I, III, X) healthy 


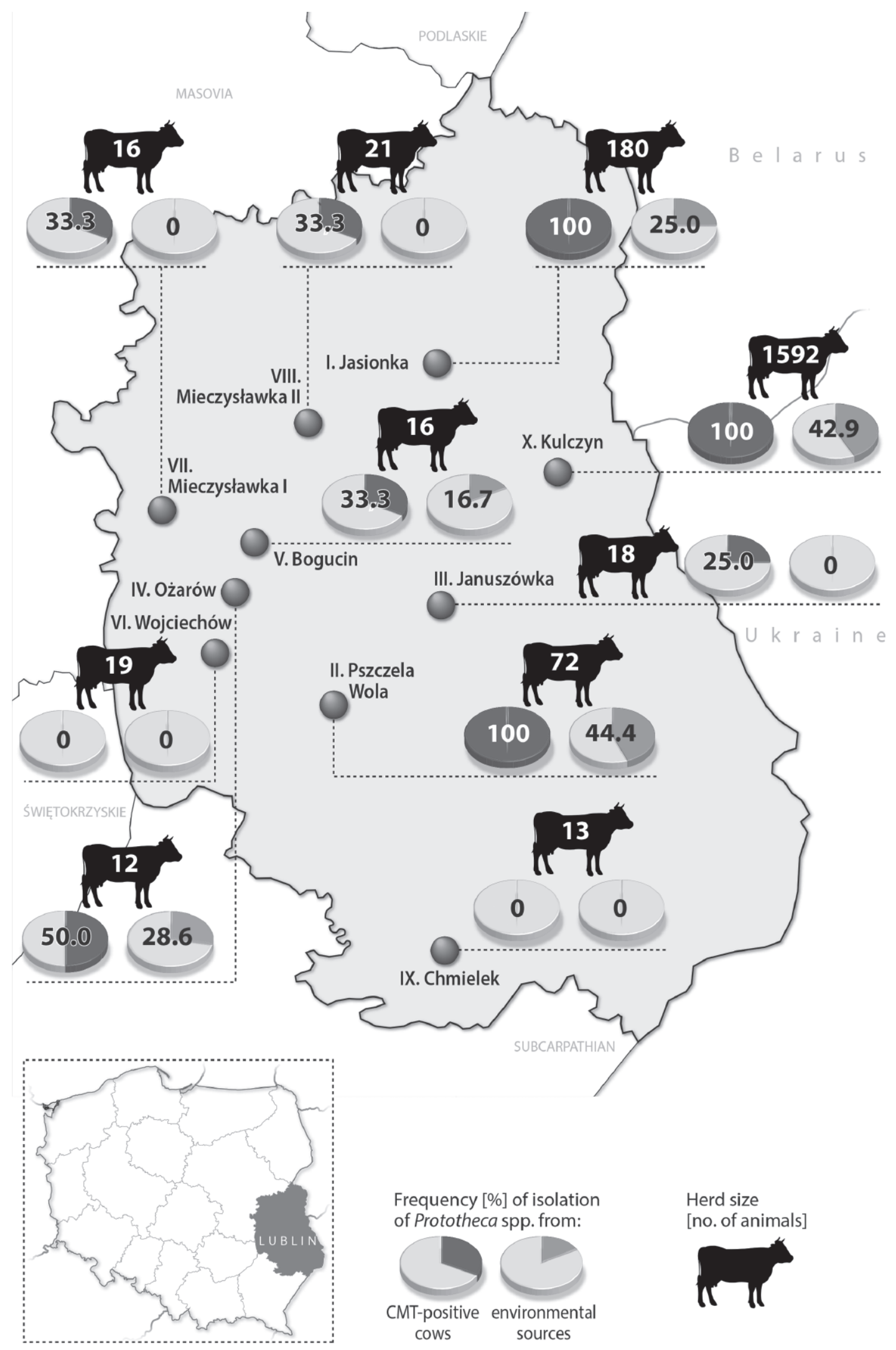

Figure 1. Prevalence of Prototheca spp. in California Mastitis Test (CMT)-positive cows and environmental samples collected from dairy farms (I to X) in Lublin province, Poland. 
cows (i.e., clinically normal and CMT-negative) were sampled for quarter milk, whole blood, feces, and oral, nasal, rectal, and vaginal swabs.

Cows whose milk yielded Prototheca spp. in culture were revisited and sampled for the same specimens as the healthy animals. Milk samples were collected aseptically, according to the National Mastitis Council guidelines (National Mastitis Council, 2017). Briefly, the teats were washed, dried, and disinfected. After discarding the first 3 streams, the milk $(2-4 \mathrm{~mL})$ was collected into sterile tubes, with a separate tube for each udder fraction. All samples of the remaining types, including environmental samples, were collected into sterile vials or containers. All samples were transported, under refrigeration $\left(4^{\circ} \mathrm{C}\right)$, to the laboratory for microbiological analyses. Blood samples were additionally subjected to standard hematological analysis.

Environmental sources sampled included water (tap water, drinking water from watering troughs, sewage, puddles, and any other watercourses on farms in their vicinity), surficial sources (topsoil, mud), plant sources (e.g., vegetation surrounding animal lots, plant compost), and other sources (animal feed/feeders/bedding, dung channels, milking machines).

\section{Primary Isolation and Culture}

All animal samples were cultivated in Columbia Agar with 5\% sheep blood (Oxoid/Thermo Fisher, Waltham, MA), McConkey agar (Sigma Aldrich, St. Louis, MO), Sabouraud dextrose agar (SDA, Difco Laboratories, Detroit, MI), Edwards's Lab-Agar (Biocorp, Warsaw, Poland), and Prototheca Isolation Medium (PIM; Pore, 1973), under aerobic conditions at $37^{\circ} \mathrm{C}$ for up to $96 \mathrm{~h}$. Environmental samples were cultured in PIM only. Liquid and semisolid specimens were spread on the medium surface either directly or after dilution in sterile water. Solid specimens were ground in a sterile porcelain vessel with sterile water, and then plated as above. Swabs from farm facilities and equipment were directly streaked on agar plates. To enhance the detection and recovery of algae, the samples were enriched with liquid PIM and cultured for $48 \mathrm{~h}$ at $37^{\circ} \mathrm{C}$ before plating.

All isolates used in the study are preserved (and available upon request) in the Culture Collection of the Department of Applied Microbiology, Faculty of Biology, University of Warsaw, Poland.

\section{Species Identification}

Any grown colonies were subjected to species identification, based on macro- and micromorphological criteria, using standard staining procedures. A milk sample was classified as positive for Staphylococcus aureus or Streptococcus agalactiae when at least one characteristic colony was identified. Other microorganisms were considered as etiological agents of mastitis if more than 3 colonies were observed. Preliminary identification included colony morphology assessment, hemolytic profiling, and Gram staining (Color Gram 2 kit, bioMérieux, Marcy l'Étoile, France). For grampositive cocci, a catalase test was carried out to differentiate catalase-negative streptococci and enterococci from catalase-positive staphylococci. Identification as Staph. aureus was based on conventional biochemical tests: catalase and coagulase reactivity, and clumping factor production using latex agglutination assay (Slidex Staph Kit, bioMérieux). A positive Christie, Atkins, Munch-Petersen (CAMP) test and a lack of esculin hydrolysis on Edward's medium allowed differentiation of Strep. agalactiae from other streptococci (Malinowski and Kłossowska, 2002). Enterococci were initially identified with the use of bile esculin agar (Biocorp). Gram-negative bacilli were discriminated based on cultural and morphological characteristics, growth on MacConkey agar (Oxoid/Thermo Fisher), indole production, and oxidase test (bioMérieux). Coryneform bacteria (Corynebacterium bovis, Trueperella pyogenes) were primarily recognized by morphological criteria. Presumptive yeast colonies were subcultured on SDA and confirmed microscopically.

\section{Prototheca spp. Identification and Typing}

Colonies suspected of being Prototheca spp. were subcultured on SDA or PIM plates and subjected to initial species identification. This included macro- and microscopic evaluation of colony morphology, and carbohydrate assimilation profiling, with the API 20C AUX system (bioMérieux). Phenotype-based identification was then confirmed with molecular methods. For these to be applied, DNA extraction was performed, essentially as reported elsewhere (Jagielski et al., 2017b). Species- and genotype-level identification was carried out using genotype-specific PCR assays, as described by Roesler et al. (2006) and with a newly described PCR-restriction enzyme analysis (PCR-REA) assay of partial cytB gene, as proposed by Jagielski et al. (2018).

All Prototheca isolates, under appropriate species (genotype) assignations were preserved in Viabank vials (Medical Wire \& Equipment Co Ltd., Corsham, $\mathrm{UK}$ ) and stored at $-70^{\circ} \mathrm{C}$.

\section{Statistical Analysis}

The Mann-Whitney U-test for independent means was used to evaluate differences between SCC and 
blood cell counts of mastitis and healthy cows. Significance was defined at $P<0.05$.

\section{RESULTS}

\section{Farm Management}

The dairy farms investigated in this study are shown in Figure 1 and their characteristics are given in Supplemental Table S1 (https://doi.org/10.3168/jds .2018-15495). They all housed Holstein-Friesian dairy cows, except 2 farms with Montbéliarde (I) and Polish White-Backed (VII) cattle. The mean herd size was 100 (range, 12-806). Most of the dairy farms had an open herd turnover $(60 \%)$, tiestall barns $(80 \%)$, with straw bedding (100\%), traditional (80\%) feeding, and drinking water supplied by municipal water distribution network $(100 \%)$. The cows had temporary access to pasture and grazing grass $(60 \%)$ and were under regular veterinary inspection (80\%). The average 305-d milk production was 7,087 L per cow (range, 6,000-10,794 L), and the mean culling rate was $24.7 \%$ (range, 20-42\%), with the predominant reasons for culling being infertility and mastitis.

The agricultural and zoohygienic (maintenance conditions affecting health and productivity of animals) aspects of the dairy cattle breeding in farms under the study are presented in Supplemental Table S1 (https://doi.org/10.3168/jds.2018-15495).

On all farms, milking was done mechanically in a double-row milking parlor $(20 \%)$ or with a milking pipeline (80\%), twice daily by experienced dairy farm milkers. On all farms, the milking staff used aprons and gloves on a regular basis. Premilking udder preparation involved washing teats by hand with water, drying teats with a paper towel, and disinfecting teats just before the machine attachment. A common (9/10 farms) postmilking hygiene practice was teat dipping in various iodine-based, commercially available products.

\section{Identification of Udder Infection}

Among all herds in the study, 100 dairy cows out of $172(58.1 \%)$ tested positive by CMT. The proportion of CMT-positive cows in a sampled herd ranged from $37.5 \%$ (farm VI) to $85.7 \%$ (farm I), with a mean of $57.1 \%$ (Figure 2; Supplemental Table S2; https://doi .org/10.3168/jds.2018-15495).

Of the 179 QMS taken from CMT-positive cows, 114 (63.7\%) QMS from 71 (71\%) cows yielded microbial growth in culture. The relative culture-positive ratio differed between farms, ranging from $23.5 \%$ (farm VII) to $60 \%$ (farms III and X).
In all but 1 (VII) dairy herds, at least one culture of Streptococcus spp. was recovered from milk samples (50 cultures from 33 cows, in total). In all but 2 (VII and IX) dairy herds, at least one Staphylococcus culture was obtained from milk samples (45 cultures from 34 cows). Isolates of Enterobacteriaceae and Pseudomonas spp. were grown from single samples collected in $2(\mathrm{~V}$ and $\mathrm{X}$ ) and 1 (IX) herd, respectively. Yeast species were cultured from 1 (I and X), 2 (III), and 3 (V) samples, each representing individual cows. Overall, bacterial microflora was isolated from 110 (61.5\%) QMS from 66 (66\%) CMT-positive cows, resulting in a total incidence of $38.4 \%$. Streptoccoci and staphylococci predominated, being almost equally represented [50 $(27.9 \%)$ vs. 45 or $(25.1 \%)]$.

\section{Protothecal Mastitis}

Cows with protothecal mastitis were from 7 dairy farms (II-V, VII, VIII, and X). Clinical and subclinical disease was recognized on 2 (II and VII) and 5 (III-V, VIII, and X) farms, respectively. These were all single cases ( 1 case per herd), except farm IV, where 2 cows were affected. The prevalence of protothecal mastitis in individual herds ranged from $0 \%$ (farms I, VI, and IX) to $25 \%$ (IV) with a mean of $4.6 \%$.

Prototheca spp. were detected in 13 (5.6\%) QMS collected from 8 cows housed in 7 dairy farms (II-V, VII, VIII, and X). Two of these cows experienced clinical changes in their udders. In milk of $5(62.5 \%)$ cows, Prototheca spp. comprised the only microflora cultured. In $3(37.5 \%)$ SCM cows, Prototheca spp. grew along with, but never from the same QMS, staphylococci (2 cows from herds IV and X) or staphylococci and yeasts (a cow from herd V).

The average SCC of the Prototheca-containing milk was $4.02 \times 10^{6}$ cells $/ \mathrm{mL}$ compared with $0.13 \times 10^{6}$ cells $/ \mathrm{mL}$ in the Prototheca-free milk collected from control animals $(P<0.00001)$. No significant differences between SCC of milk samples from CM and SCM cows were observed (mean counts $4.59 \times 10^{6}$ and 3.45 $\times 10^{6}$ cells $/ \mathrm{mL}$ ). Likewise, we detected no significant differences between mastitis and control cows with respect to counts of total white blood cells, lymphocytes, neutrophils, and eosinophils (Table 1).

\section{Prototheca spp. in Stool and Body Site Swabs}

Overall, 151 body site swabs and stool samples were collected from 23 healthy cows and 8 cows with protothecal mastitis. Among 19 Prototheca spp. cultures, 14 were isolated from 8 healthy cows, whereas the remaining 5 were isolated from cows with SCM (4) and CM 
Table 1. Milk SCC and blood cell counts $( \pm \mathrm{SD})$ in cows with clinical and subclinical Prototheca mastitis and in control cows

\begin{tabular}{lccc}
\hline Item & $\begin{array}{c}\text { Clinical mastitis } \\
\left(\mathrm{n}=5 \mathrm{QMS}^{1}\right)\end{array}$ & $\begin{array}{c}\text { Subclinical mastitis } \\
(\mathrm{n}=8 \text { QMS })\end{array}$ & $\begin{array}{c}\text { Control } \\
(\mathrm{n}=92 \text { QMS })\end{array}$ \\
\hline Average milk SCC, $\times 10^{6} / \mathrm{mL}$ & $4.59 \pm 3.61$ & $3.45 \pm 2.03$ & $0.13 \pm 0.01$ \\
White blood cells, $^{2} \times 10^{9} / \mathrm{L}$ & $7.75 \pm 2.55$ & $6.10 \pm 0.70$ & $7.13 \pm 1.40$ \\
Lymphocytes $^{2} \times 10^{9} / \mathrm{L}$ & $3.55 \pm 1.45$ & $3.02 \pm 0.56$ & $3.77 \pm 1.03$ \\
Neutrophils, $^{2} \times 10^{9} / \mathrm{L}$ & $2.65 \pm 1.45$ & $2.05 \pm 0.53$ & $2.33 \pm 1.03$ \\
Eosinophils, $^{2} \times 10^{9} / \mathrm{L}$ & $1.25 \pm 0.55$ & $0.72 \pm 0.18$ & $0.74 \pm 0.55$ \\
Cows, no. $(\%)^{3}$ & $2(1.11)$ & $6(3.34)$ & $23(12.85)$ \\
Herds, no. $(\%)$ & $2(20)$ & $5(50)$ & $10(100)$ \\
\hline
\end{tabular}

${ }^{1}$ Quarter milk sample.

${ }^{2}$ Average counts for all animals from each group.

${ }^{3}$ Percentage of cows of different groups given in parentheses.

(1). Prototheca spp. collected from the affected animals were found in stool (4 isolates; 1 from $\mathrm{CM}$ and 3 from SCM cows) and vagina (1 isolate from SCM cow). A wider distribution occurred among isolates cultured from control animals. Here, Prototheca spp. were isolated from mouth (3), nose (3), vagina (1), rectum (3), and feces (4) (Figure 2; Supplemental Table S2; https: //doi.org/10.3168/jds.2018-15495).

A

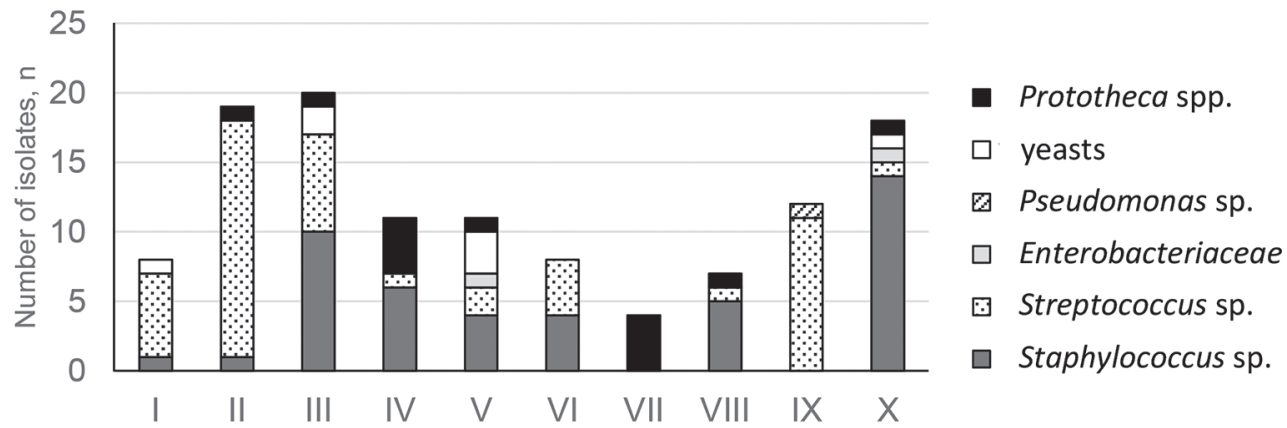

B

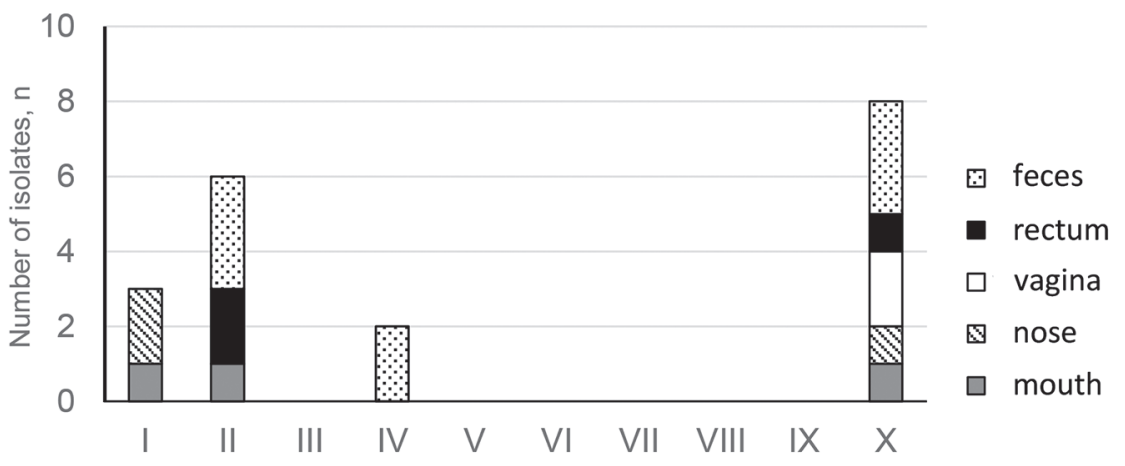

C



Figure 2. Distribution of mastitis pathogens in milk samples (A) and Prototheca spp. in body swabs (B) and environmental sources (C) in dairy farms (I to X) in Lublin province, Poland. 


\section{Prototheca spp. in Environmental Samples}

A total of 91 environmental samples were collected (Supplemental Table S2; https://doi.org/10.3168/jds $.2018-15495)$. Of these, $12(13.2 \%)$ were positive for Prototheca spp. These samples originated from 5 dairy farms (I, II, IV, V, and X), including 1 (I) where no Prototheca spp. were isolated from milk samples. Among the environmental sites that yielded Prototheca growth were watering trough (5 positive samples), manure (4), feed (2), and mud (1).

\section{Prototheca spp. Genotyping}

All (44) Prototheca isolates recovered in this study were subjected to species- and genotype-level molecular identification (Figure 3; Supplemental Table S3; https: //doi.org/10.3168/jds.2018-15495). Milk-derived isolates belonged exclusively to $P$. zopfii gen. 2. Among 20 isolates from animal swabs and stool, 15 isolates were P. zopfii gen. 2, 2 isolates were P. zopfii gen. 1, and 2 isolates were $P$. blaschkeae. The 4 non- $P$. zopfii gen. 2 isolates were obtained from 2 fecal samples from 2 cows with subclinical mastitis from dairy farm IV ( $P$. zopfii gen. 1) and from rectum and feces of 2 control cows from dairy farm X. Of 12 environmental isolates, none were $P$. blaschkeae, 4 were $P$. zopfii gen. 1 , and 8 were P. zopfii gen. 2.

\section{DISCUSSION}

The results from this study show that Prototheca spp. are the third most common cause of mastitis in dairy cows in the population we studied, after streptococci and staphylococci. Similar findings; that is, placing Prototheca algae among the top 3 etiological agents of bovine mastitis, have been reported in the Piedmont region of Italy; Ontario, Canada; and São Paulo State, Brazil (Costa et al., 1996; Buzzini et al., 2004; Pieper et al., 2012).

At the herd level, the prevalence of protothecal mastitis was within the range of 0 to $25 \%$, similar to that described in the eastern United States, Brazil, Serbia and Montenegro, Japan, and Italy (Pore et al., 1987; Corbellini et al., 2001; Milanov et al., 2006; Osumi et al., 2008; Arrigioni et al., 2010; Bozzo et al., 2014). It should be noted here that our rates may be somewhat underestimated, given that in 2 herds (I and X), only a proportion of cows (48.2 and 3.7\%, respectively) were examined. In the remaining herds (II-IX), an average of $83 \%$ of cows were examined (range, 66.7-95.5\%).
Only a single herd had more than one Prototheca mastitis case confirmed (herd IV, 2 cows with SCM), thus being indicative of an outbreak. There have been a few reports of mastitis outbreaks due to Prototheca spp., involving 5 to 34 animals, depending on the herd size (average of 13 mastitis animals per herd; Costa et al., 1996; Abarca et al., 2001; Bueno et al., 2006; Gao et al., 2012; Ricchi et al., 2013; Wawron et al., 2013).

Protothecal bovine mastitis predominantly follows a subclinical or chronic course (Costa et al., 1996; Abarca et al., 2001; Bueno et al., 2006; Wawron et al., 2013). Based on clinical examination, SCC, and Prototheca spp. culture from QMS, $2(1.2 \%)$ and $6(3.5 \%)$ cows were classified as suffering from CM and SCM, respectively. According to previous studies, the number of cows with protothecal SCM was much higher than that of cows with CM (Abarca et al., 2001; Bueno et al., 2006). A predominance of CM cases was also documented (Gao et al., 2012; Ricchi et al., 2013).

In this study, SCC were only slightly higher in $\mathrm{CM}$ than in SCM (4.59 vs. $3.45 \times 10^{6}$ cells $\left./ \mathrm{mL}\right)$, although other studies have reported SCC approximately 10-fold higher for CM cases (Malinowski and Kłossowska, 2002; Jagielski et al., 2011). The algae were also isolated from the QMS with physiological SCC; that is, $>10^{5}$ cells $/ \mathrm{mL}$ (data not shown). An elevated level of SCC $(>200 \times$ $10^{3}$ cells $/ \mathrm{mL}$ ) is frequently observed in SCM, although it is not always mandatory for the diagnosis (Tenhagen et al., 2005; Bueno et al., 2006; Bozzo et al., 2014).

Interestingly, no significant changes in blood cell counts were found between mastitis and control cows or between SCM and CM animals. This is in contrast with what was observed by Alhussien et al. (2015), where total leukocyte counts of CM cows were higher than those in SCM cows.

Half of the cows with Prototheca spp. (4\% of all CMT-positive cows) in their milk did not yield Prototheca from other anatomical sites. In 8 cows $(8 \%$ of all CMT-positive cows) whose milk was negative for Prototheca spp., the algae could be cultured from feces (4 cows), mouth, nose, rectum (3 cows each), and vagina (one cow). This clearly shows that Prototheca spp. are colonizers of the bovine gastrointestinal and urogenital tract without causing any harm to the host. The asymptomatic carrier state of the algae has already been documented in dairy herds (Tenhagen et al., 2005; Bueno et al., 2006).

Finding Prototheca spp. in different environmental sites indicates their relatively wide distribution in nature, especially in humid and wet areas containing manure and decomposing plant material (Milanov et al., 2016). In this study, the algae were found mainly 
A



B
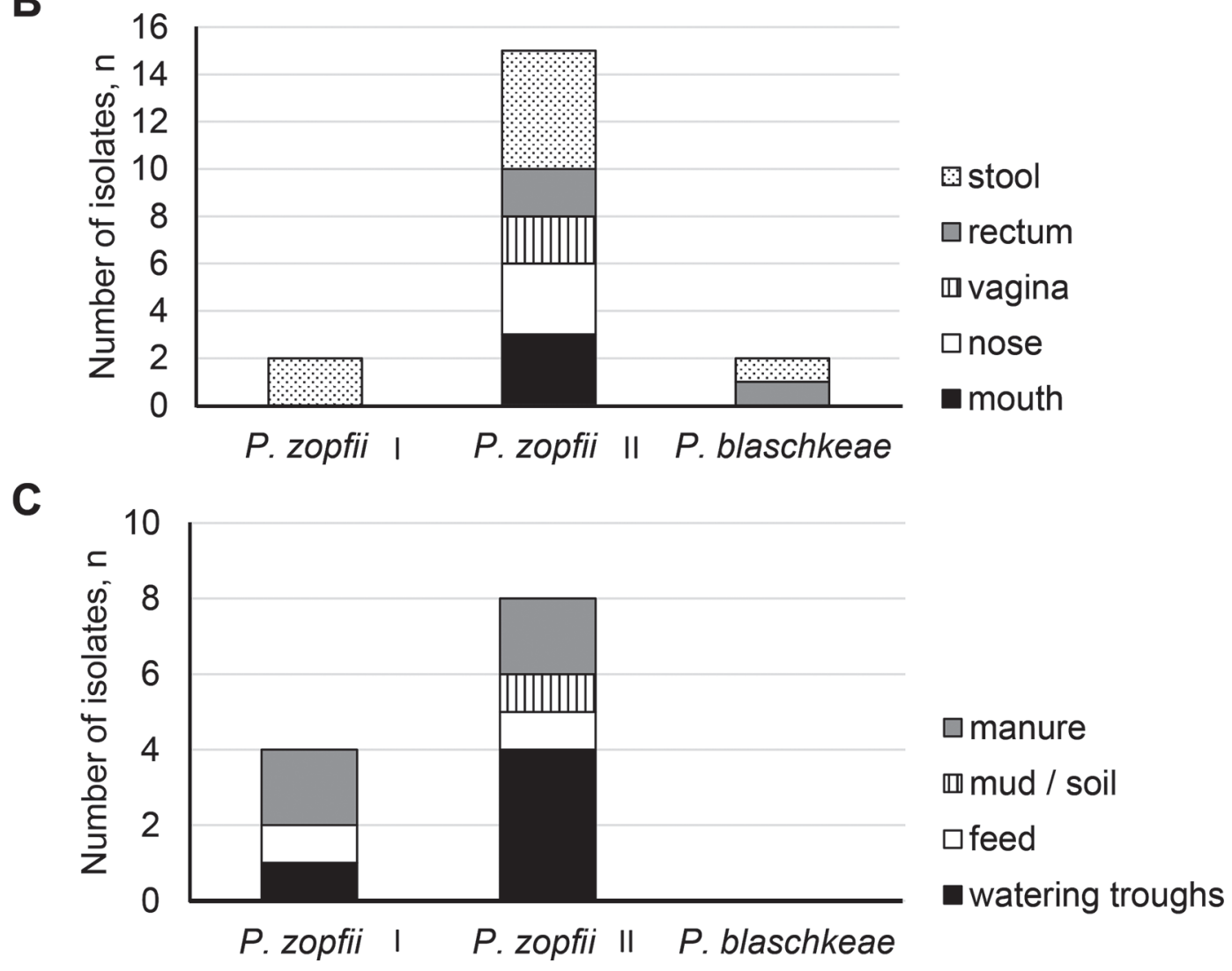

Figure 3. Species- and genotype-level identification of Prototheca isolates from this study in milk samples (A), body swabs (B), and environmental sources $(\mathrm{C})$. QMS $=$ quarter milk sample.

in drinking water and manure, and these sources have been their major environmental reservoir, as reported in previous studies (Pore et al., 1983; Enders and Weber, 1993; Costa et al., 1997; Scaccabarozzi et al., 2008).

At the species and genotype level, only P. zopfii gen. 2 was identified in milk samples, confirming its predominant role as a causative agent in protothecal mastitis in cattle (Osumi et al., 2008; Ahrholdt and Rosler, 2011; Jagielski et al., 2011; Pieper et al., 2012). Prototheca zopfii gen. 2 was also recovered from most (80\%) of the body site swabs, with only $2(10 \%)$ samples yielding $P$. blaschkeae. The recovery of Prototheca spp. from environmental samples indicates their persistence in the dairy herd ecosystem.

The present work is a prelude to a whole-country investigation currently underway of the epidemiology of Prototheca bovine mastitis. Nevertheless, this study provides, for the first time in Poland, a comprehensive 
and informative look at the occurrence of Prototheca on the regional scale.

\section{ACKNOWLEDGMENTS}

The study was financed by the National Science Centre "SONATA" Programme (Krakow, Poland; 2014/15/D/NZ7/01797).

\section{REFERENCES}

Aalbaek, B., H. E. Jensen, and A. Huda. 1998. Identification of Prototheca from bovine mastitis in Denmark. APMIS 106:483-488.

Abarca, M. L., L. Ruano, E. Torre, and F. J. Cabañes. 2001. Subclinical bovine mastitis by Prototheca zopfii in Spain. J. Mycol. Med. 11:2.

Ahrholdt, J., and U. Rosler. 2011. Genotypical differentiation of Prototheca isolates of milk samples from mastitis affected cattle in Germany. Berl. Munch. Tierarztl. Wochenschr. 124:108-113.

Alhussien, M., M. Kaur, P. Manjari, S. P. Kimothi, A. K. Mohanty, and A. K. Dang. 2015. A comparative study on the blood and milk cell counts of healthy, subclinical, and clinical mastitis Karan Fries cows. Vet. World 8:685-689.

Amer, S., F. L. A. Galvez, Y. Fukuda, C. Tada, I. L. Jimenez, W. F. M. Valle, and Y. Nakai. 2018. Prevalence and etiology of mastitis in dairy cattle in El Oro Province, Ecuador. J. Vet. Med. Sci. 80:861-868.

Anderson, K. L., and R. L. Walker. 1988. Sources of Prototheca spp. in a dairy-herd environment. J. Am. Vet. Med. Assoc. 193:553-556.

Arrigioni, N., G. L. Belletti, G. Cammi, C. Garbarino, and M. Ricchi. 2010. Prototheca bovine mastitis. Large Anim. Rev. 16:5.

Bozzo, G., E. Bonerba, A. Di Pinto, G. Bolzoni, E. Ceci, A. Mottola, G. Tantillo, and V. Terio. 2014. Occurrence of Prototheca spp. in cow milk samples. New Microbiol. 37:459-464.

Bueno, V. F., A. J. de Mesquita, R. B. Neves, M. A. de Souza, A. R Ribeiro, E. S. Nicolau, and A. N. de Oliveira. 2006. Epidemiological and clinical aspects of the first outbreak of bovine mastitis caused by Prototheca zopfii in Goias State, Brazil. Mycopathologia 161:141-145.

Buzzini, P., B. Turchetti, R. Facelli, R. Baudino, F. Cavarero, L. Mattalia, P. Mosso, and A. Martini. 2004. First large-scale isolation of Prototheca zopfii from milk produced by dairy herds in Italy. Mycopathologia 158:427-430.

Corbellini, L. G., D. Driemeier, C. Cruz, M. M. Dias, and L. Ferreiro. 2001. Bovine mastitis due to Prototheca zopfii: Clinical, epidemiological and pathological aspects in a Brazilian dairy herd. Trop. Anim. Health Prod. 33:463-470.

Costa, E. O., A. C. Carciofi, P. A. Melville, M. S. Prada, and U. Schalch. 1996. Prototheca sp. outbreak of bovine mastitis. Zentralbl. Veterinarmed. B 43:321-324.

Costa, E. O., P. A. Melville, A. R. Ribeiro, E. T. Watanabe, and M. C. Parolari. 1997. Epidemiologic study of environmental sources in a Prototheca zopfii outbreak of bovine mastitis. Mycopathologia 137:33-36.

De Vliegher, S., L. K. Fox, S. Piepers, S. McDougall, and H. W. Barkema. 2012. Invited review: Mastitis in dairy heifers: Nature of the disease, potential impact, prevention, and control. J. Dairy Sci. 95:1025-1040.

Enders, F., and A. Weber. 1993. The occurrence of Prototheca in fecal samples of cattle. Berl. Munch. Tierarztl. Wochenschr. 106:165169.

Gao, J., H. Q. Zhang, J. Z. He, Y. H. He, S. M. Li, R. G. Hou, Q. X. Wu, Y. Gao, and B. Han. 2012. Characterization of Prototheca zopfii associated with outbreak of bovine clinical mastitis in herd of Beijing, China. Mycopathologia 173:275-281.
Gonzalo, C., J. A. Baro, J. A. Carriedo, and F. S. Primitivo. 1993. Use of the Fossomatic method to determine somatic cell counts in sheep milk. J. Dairy Sci. 76:115-119.

Hiitiö, H., J. Vakkamäki, H. Simojoki, T. Autio, J. Junnila, S. Pelkonen, and S. Pyörälä. 2017. Prevalence of subclinical mastitis in Finnish dairy cows: Changes during recent decades and impact of cow and herd factors. Acta Vet. Scand. 59:22.

Ito, T., R. Kano, H. Sobukawa, J. Ogawa, Y. Honda, Y. Hosoi, H. Shibuya, T. Sato, A. Hasegawa, and H. Kamata. 2011. Experimental infection of bovine mammary gland with Prototheca zopfii genotype 1. J. Vet. Med. Sci. 73:117-119.

Jagielski, T., Z. Bakuła, S. Di Mauro, C. Casciari, V. Cambiotti, H. Krukowski, B. Turchetti, M. Ricchi, E. Manuali, and P. Buzzini. 2017a. A comparative study of the in vitro activity of iodopropynyl butylcarbamate and amphotericin B against Prototheca spp. isolates from European dairy herds. J. Dairy Sci. 100:7435-7445.

Jagielski, T., P. Buzzini, H. Lassa, E. Malinowski, E. Branda, B. Turchetti, A. Polleichtner, U. Roesler, P. E. Lagneau, S. Marques, E. Silva, G. Thompson, R. Stachowiak, and J. Bielecki. 2012. Multicentre Etest evaluation of in vitro activity of conventional antifungal drugs against European bovine mastitis Prototheca spp. isolates. J. Antimicrob. Chemother. 67:1945-1947.

Jagielski, T., J. Gawor, Z. Bakuła, P. Decewicz, K. Maciszewski, and A. Karnkowska. 2018. cytb as a new genetic marker for differentiation of Prototheca species. J. Clin. Microbiol. 56:e00584-18. https: //doi.org/1128/JCM.00584-18.

Jagielski, T., J. Gawor, Z. Bakuła, K. Zuchniewicz, I. Żak, and R. Gromadka. 2017b. An optimized method for high quality DNA extraction from microalga Prototheca wickerhamii for genome sequencing. Plant Methods 13:77.

Jagielski, T., H. Lassa, J. Ahrholdt, E. Malinowski, and U. Roesler. 2011. Genotyping of bovine Prototheca mastitis isolates from Poland. Vet. Microbiol. 149:283-287.

Kirkpatrick, M. A., and J. D. Olson. 2015. Somatic cell counts at first test: More than a number. Pages 53-56 in Proc. NMC Annual Meeting, Memphis, TN. National Mastitis Council (NMC) Inc., Madison, WI.

Lagneau, P. E. 1996. First isolation of Prototheca zopfii in bovine mastitis in Belgium. J. Mycol. Med. 6:145-148.

Lerche, M. 1952. Eine durch Algen (Prototheca) hervorgerufene Mastitis der Kuh. Berl. Munch. Tierarztl. Wochenschr. 4:64-69.

Malinowski, A., and A. Kłossowska. 2002. Diagnostics of intramammary infections. National Veterinary Research Institute, Puławy, Poland.

Malinowski, E., H. Lassa, and A. Kłossowska. 2002. Isolation of Prototheca zopfii from inflamed secretion of udders. B. Vet. I. Pulawy $46: 295-299$

Marques, S., E. Silva, C. Kraft, J. Carvalheira, A. Videira, V. A. Huss, and G. Thompson. 2008. Bovine mastitis associated with Prototheca blaschkeae. J. Clin. Microbiol. 46:1941-1945.

Milanov, D., T. Petrović, V. Polaček, L. Suvajdžić, and J. Bojkovski. 2016. Mastitis associated with Prototheca zopfii-An emerging health and economic problem on dairy farms. J. Vet. Res. (Pulawy) 60:373-378.

Milanov, D., L. Suvajdžić, I. Pušić, B. Vidić, and V. Milic Torres. 2006. Outbreak of endemic form of protothecal mastitis on a dairy farm. Acta Vet. (Beogr.) 56:7.

Möller, A., U. Truyen, and U. Roesler. 2007. Prototheca zopfii genotype 2 - The causative agent of bovine protothecal mastitis? Vet. Microbiol. 120:370-374.

National Mastitis Council. 2017. Laboratory Handbook on Bovine Mastitis. National Mastitis Council Inc., Madison, WI.

Osumi, T., Y. Kishimoto, R. Kano, H. Maruyama, M. Onozaki, K. Makimura, T. Ito, K. Matsubara, and A. Hasegawa. 2008. Prototheca zopfii genotypes isolated from cow barns and bovine mastitis in Japan. Vet. Microbiol. 131:419-423.

Pieper, L., A. Godkin, U. Roesler, A. Polleichtner, D. Slavic, K. E. Leslie, and D. F. Kelton. 2012. Herd characteristics and cow-level factors associated with Prototheca mastitis on dairy farms in Ontario, Canada. J. Dairy Sci. 95:5635-5644. 
Pore, R. S. 1973. Selective medium for the isolation of Prototheca. Appl. Microbiol. 26:648-649.

Pore, R. S., E. A. Barnett, W. C. Barnes Jr., and J. D. Walker. 1983. Prototheca ecology. Mycopathologia 81:49-62.

Pore, R. S., T. A. Shahan, M. D. Pore, and R. Blauwiekel. 1987. Occurrence of Prototheca zopfii, a mastitis pathogen, in milk. Vet. Microbiol. 15:315-323.

Quinn, P. J., M. E. Carter, B. K. Markey, and G. R. Carter. 2002. Bacterial causes of bovine mastitis. Pages 465-475 in Veterinary Microbiology and Microbial Disease. 8th ed. Mosby International Ltd., London, UK.

Ricchi, M., C. De Cicco, P. Buzzini, G. Cammi, N. Arrigoni, M. Cammi, and C. Garbarino. 2013. First outbreak of bovine mastitis caused by Prototheca blaschkeae. Vet. Microbiol. 162:997-999.

Ricchi, M., M. Goretti, E. Branda, G. Cammi, C. A. Garbarino, B. Turchetti, P. Moroni, N. Arrigoni, and P. Buzzini. 2010. Molecular characterization of Prototheca strains isolated from Italian dairy herds. J. Dairy Sci. 93:4625-4631.

Roesler, U., A. Möller, A. Hensel, D. Baumann, and U. Truyen. 2006. Diversity within the current algal species Prototheca zopfii: A pro- posal for two Prototheca zopfii genotypes and description of a novel species, Prototheca blaschkeae sp. nov. Int. J. Syst. Evol. Microbiol. $56: 1419-1425$.

Scaccabarozzi, L., B. Turchetti, P. Buzzini, G. Pisoni, L. Bertocchi, N. Arrigoni, P. Boettcher, V. Bronzo, and P. Moroni. 2008. Short communication: Isolation of Prototheca species strains from environmental sources in dairy herds. J. Dairy Sci. 91:3474-3477.

Seegers, H., C. Fourichon, and F. Beaudeau. 2003. Production effects related to mastitis and mastitis economics in dairy cattle herds. Vet. Res. 34:475-491.

Tenhagen, B. A., A. Hille, A. Schmidt, and W. Heuwieser. 2005. Shedding patterns and somatic cell counts in milk from quarters chronically infected with Prototheca spp. Dtsch. Tierarztl. Wochenschr. 112:44-48

Viguier, C., S. Arora, N. Gilmartin, K. Welbeck, and R. O'Kennedy. 2009. Mastitis detection: Current trends and future perspectives. Trends Biotechnol. 27:486-493.

Wawron, W., M. Bochniarz, T. Piech, W. Łopuszyński, and J. Wysocki. 2013. Outbreak of protothecal mastitis in a herd of dairy cows in Poland. B. Vet. I. Pulawy 57:335-339. 